\title{
Endoscopic Submucosal Dissection in Patients with Ulcerative Colitis
}

\author{
Satoshi Kinoshita ${ }^{a}$ b Toshihiro Nishizawa ${ }^{a}{ }^{a}$ Naohisa Yahagi ${ }^{b}$ \\ Toshio Uraoka ${ }^{\mathrm{a}-\mathrm{c}}$ \\ a Department of Gastroenterology, National Hospital Organization Tokyo Medical Center, Tokyo, Japan; ${ }^{b}$ Division of \\ Research and Development for Minimally Invasive Treatment, Cancer Center, Keio University School of Medicine, \\ Tokyo, Japan; 'Department of Gastroenterology and Hepatology, Gunma University Graduate School of Medicine, \\ Tokyo, Japan
}

\section{Keywords}

Endoscopic submucosal dissection - Ulcerative colitis .

Neoplasms

\section{Abstract}

Patients with ulcerative colitis have an increased risk of developing colorectal cancer. This risk has been estimated to reach about $7 \%$ at 20 years of disease, $7-14 \%$ at 25 years, and as high as $30 \%$ after 35 years. The guidelines for the management of inflammatory bowel disease recommend endoscopic resection of circumscribed dysplasia and ongoing colonoscopic surveillance as a reasonable strategy in patients with ulcerative colitis. Submucosal fibrosis due to background inflammation could hamper adequate lifting and endoscopic treatment. Endoscopic mucosal resection (EMR) is difficult for dysplasia within colitic mucosa due to the non-lifting sign. Although endoscopic submucosal dissection (ESD) generally has higher risks of perforation and bleeding compared to EMR, the technique can achieve complete en bloc resection regardless of the lesion size or severity of submucosal fibrosis. Several studies have shown that ESD for circumscribed dysplasia in ulcerative colitis is feasi- ble. While ESD can avert unnecessary surgery, submucosal fibrosis makes the intervention technically demanding in cases of ulcerative colitis. ESD should be performed by expert endoscopists using the most suitable equipment and devices available.

(c) 2018 S. Karger AG, Basel

\section{Introduction}

Patients with ulcerative colitis have an increased risk of developing colorectal cancer, a disease believed to follow the inflammation-dysplasia-carcinoma sequence. This cancer risk has been estimated to reach about $7 \%$ at 20 years of disease, $7-14 \%$ at 25 years, and as high as $30 \%$ after 35 years $[1,2]$. Restorative proctocolectomy is recommended as the standard operation when an endoscopically invisible, high-grade dysplasia is detected, as patients manifesting this symptom suffer high rates (32$42 \%)$ of either synchronous or metachronous cancer [3-5]. Proctocolectomy, however, causes frequent and watery bowel movements, as well as postoperative complications in some patients. The management of circum-

\section{KARGER}

(C) 2018 S. Karger AG, Basel

E-Mail karger@karger.com

www.karger.com/dig
Toshio Uraoka, MD, PhD

Department of Gastroenterology and Hepatology Gunma University Graduate School of Medicine

3-39-22 Showamachi, Maebashi-shi, Gunma 371-8511 (Japan)

E-Mail uraoka@gunma-u.ac.jp 
Table 1. ESD in ulcerative colitis

\begin{tabular}{llll}
\hline Author & Iacopini et al. [14] & Suzuki et al. [15] & Kinoshita et al. [18] \\
\hline Year & 2015 & 2017 & 2017 \\
Country & Italy and Japan & UK and Japan & Japan \\
Number of lesions & 10 & 32 & 25 \\
Age, years & $62(35-69)$ & $65(49-86)$ & $62(38-83)$ \\
UC duration, years & $13(9-22)$ & $20(1-41)$ & $19(1-37)$ \\
Size, mean \pm SD & $33 \pm 12$ & $33(12-73) *$ & $35 \pm 17$ \\
En bloc resection rate, \% & 80 & 91 & 100 \\
Complete rescetion rate, $\%$ & 70 & 79 & 76 \\
Bleeding rate, \% & 10 & 3 & 0 \\
Perforation rate, \% & 0 & 0 & 4 \\
Follow-up period, months & $24(6-72)$ & $33(6-76)$ & $21(8-80)$ \\
Local recurrence rate, $\%$ & 0 & 3 & 0 \\
Metachronous recurrence rate, \% & 0 & 9 & 4 \\
Additional surgery rate, \% & 0 & 13 & 20 \\
\hline
\end{tabular}

* Median (range).

ESD, endoscopic submucosal dissection.

scribed dysplastic lesions within colitic mucosa should be determined by endoscopic resectability. If the lesion is well circumscribed and can be fully removed, endoscopic resection may be an effective treatment [6-8]. Endoscopic treatment preserves the rectum and colon, which results in a better quality of life compared to that after surgery. The guidelines for the management of inflammatory bowel disease in adults recommend endoscopic resection of circumscribed dysplasia and continued colonoscopic surveillance as a reasonable strategy in patients with ulcerative colitis $[9,10]$. Endoscopic mucosal resection (EMR), however, is inadequate for the resection of dysplastic lesions within colitic mucosa [11]. Submucosal fibrosis due to background inflammation can hamper adequate lifting and endoscopic treatment [12]. The nonlifting sign makes it difficult to resect dysplasia within colitic mucosa by EMR. Piecemeal resection by EMR also raises concerns of potential recurrence or inaccurate histologic assessment due to fragmented, pathological specimens.

Endoscopic submucosal dissection (ESD) was developed in Japan in the late 1990s as an alternative method. Endoscopists initially considered ESD too risky, as the intervention had a higher risk of perforation and bleeding compared to EMR. ESD has the advantage, however, of achieving complete en bloc resection regardless of the lesion size or severity of the submucosal fibrosis [13]. The safety and success rates of ESD have improved to favorable levels in recent years. Given the great merit of ESD and safety improvements, the technique has been adopt- ed as a standard treatment in Japan and other East Asian countries. Several reports have also suggested that ESD is feasible for large neoplasms in ulcerative colitis [14-16]. In this article, we review ESD as a treatment for neoplastic lesions in ulcerative colitis.

\section{ESD in Ulcerative Colitis}

Iacopini et al. [14] assessed the feasibility and efficacy of ESD for ulcerative colitis. Endoscopically invisible dysplasia and endoscopically visible moderate-to-severe active ulcerative colitis were excluded. A total of 10 superficial non-polypoid neoplasms of $>20 \mathrm{~mm}$ (mean size $33 \pm 12 \mathrm{~mm}$ ) within the colitic mucosa were included (7 in Italian patients, 3 in Japanese patients). Submucosal fibrosis was present in $90 \%(9 / 10)$ of the cases. The en bloc and complete resection rates were $80 \%(8 / 10)$ and $70 \%$ $(7 / 10)$, respectively. Complete resection was defined as en bloc resection with free lateral and vertical margins. Delayed bleeding occurred in $10 \%(1 / 10)$ of the patients. No local recurrences or metachronous lesions appeared over a median follow-up period of 24 months (Table 1). A metachronous lesion was defined as a new lesion detected more than 1 year following treatment.

Suzuki et al. [15] also assessed the feasibility of ESD for ulcerative colitis. They examined 32 dysplastic lesions in total (23 in British patients, 9 in Japanese patients), after excluding lesions with severely inflamed background mucosa and no discernible demarcation lines. The 32 le- 
sions examined had a median size of $33 \mathrm{~mm}$ (range 12-73 $\mathrm{mm})$, and $97 \%(31 / 32)$ of them manifested submucosal fibrosis. The rates of en bloc and complete resection were 91\% (29/32) and 79\% (25/32), respectively. Delayed bleeding occurred in 3\% (1/32) of the patients. Four patients, including 2 with T1 cancer, one with invisible dysplasia, and one with mild dysplasia who preferred to undergo surgery, subsequently underwent panproctocolectomy. The rates of local recurrence and metachronous lesions were $3 \%(1 / 32)$ and $10 \%(3 / 32)$, respectively, over a median follow-up period of 33 months.

Kinoshita et al. [16] reported short- and long-term outcomes of ESD for ulcerative colitis. They examined 25 dysplastic lesions at Keio University Hospital and Tokyo Medical Center, excluding patients with endoscopically invisible dysplasia and endoscopic moderate-to-severe active ulcerative colitis. The lesions had a mean size of $35 \pm 17 \mathrm{~mm}$. The rates of en bloc and complete resection were $100 \%(25 / 25)$ and $76 \%$ (19/25), respectively. Perforation during ESD occurred in $4 \%(1 / 25)$ of the patients. Non-curative resection due to submucosal invasion $\geq 1,000 \mu \mathrm{m}$ or lymphovascular involvement was seen in $20 \%(5 / 25)$ of the patients, all of whom went on to undergo additional surgery. The rates of local recurrence and metachronous high-grade dysplasia were $0 \%$ and $4 \%$ $(1 / 25)$, respectively, over a median follow-up period of 21 months.

\section{Preoperative Diagnoses in Ulcerative Colitis}

Discrepancies between biopsy and histological diagnoses of resected specimens have been reported for colorectal lesions. The sensitivity of biopsy was reported to be $83.6 \%$ [17]. The discrepancies are likely to reflect the heterogeneity of these tumors, as the tumors are thought to follow the adenoma-carcinoma sequence in the duodenum, colon, and rectum [18]. Our group previously assessed the sensitivity of preoperative diagnoses based on biopsy in ulcerative colitis [16]. Biopsy predicted a final histologic diagnosis of carcinoma with a sensitivity of $72.2 \%(13 / 18)$ and accuracy of $78.2 \%$ (18/23). Our previous study showed a relatively low sensitivity and accuracy of biopsy in patients with ulcerative colitis. Targeted biopsies from depressed or reddish areas may minimize the underestimation of biopsy diagnoses.

Discrepancies between endoscopic findings and histological diagnoses of resected specimens have also been reported for colorectal lesions. Early colorectal adenocarcinomas are differentiated from adenomas with a re- ported sensitivity and accuracy of $83-85$ and $73 \%$, respectively, in magnifying endoscopy with crystal violet dye or magnifying narrow-band imaging (NBI) [19]. Our group previously assessed the sensitivity and accuracy of endoscopic preoperative diagnoses in ulcerative colitis [16]. The endoscopic findings (crystal violet staining and magnifying NBI) predicted a final histologic diagnosis of carcinoma or high-grade dysplasia with a sensitivity of $72.2 \%(13 / 18)$ and accuracy of $76 \%(19 / 25)$. Endoscopists should be aware of this rather low sensitivity and tendency towards underestimation in endoscopic diagnosis. The biopsy procedure itself may induce unexpected fibrosis, which may complicate subsequent endoscopic treatment. Establishing a pre-operative diagnostic method without biopsy would be helpful. A new endoscopic modality that improves preoperative diagnosis is awaited.

\section{Case Presentation}

\section{Case 1}

The patient was a 42 -year-old male with a 19 -year history of left-sided ulcerative colitis treated with 5-aminosalicylic acid. A laterally spreading lesion of $35 \mathrm{~mm}$ in diameter was discovered in the patient's lower rectum. The capillary pattern was Type 2B in the Japanese NBI Expert Team (JNET) classification. The pit pattern in crystal violet staining was Type $\mathrm{V}_{\mathrm{I}}$-low in the Kudo classification. The preoperative diagnosis based on the Type 2B JNET classification and $\mathrm{V}_{\mathrm{I}}$-low pit pattern was intramucosal cancer (high-grade dysplasia). Submucosal dissection was carefully conducted after injecting sodium hyaluronate solution (Fig. 1). Histological examination of the resected specimen revealed well-differentiated mucosal adenocarcinoma without lymphatic or vascular involvement. Severe fibrosis was observed in the submucosal layer, but the vertical and horizontal margins were both negative.

\section{Case 2}

The patient was a 65 -year-old male with a 24 -year history of left-sided ulcerative colitis treated with an immunomodulatory drug and 5-aminosalicylic acid. An $11-\mathrm{mm}$ laterally spreading tumor was found in the patient's rectum. The capillary pattern was Type $2 \mathrm{~B}$ of the JNET classification. The pit pattern in crystal violet staining was Type $\mathrm{V}_{\mathrm{I}}$-low in the Kudo classification. The preoperative diagnosis based on the Type 2B JNET classification and $\mathrm{V}_{\mathrm{I}}$-low pit pattern was intramucosal can- 

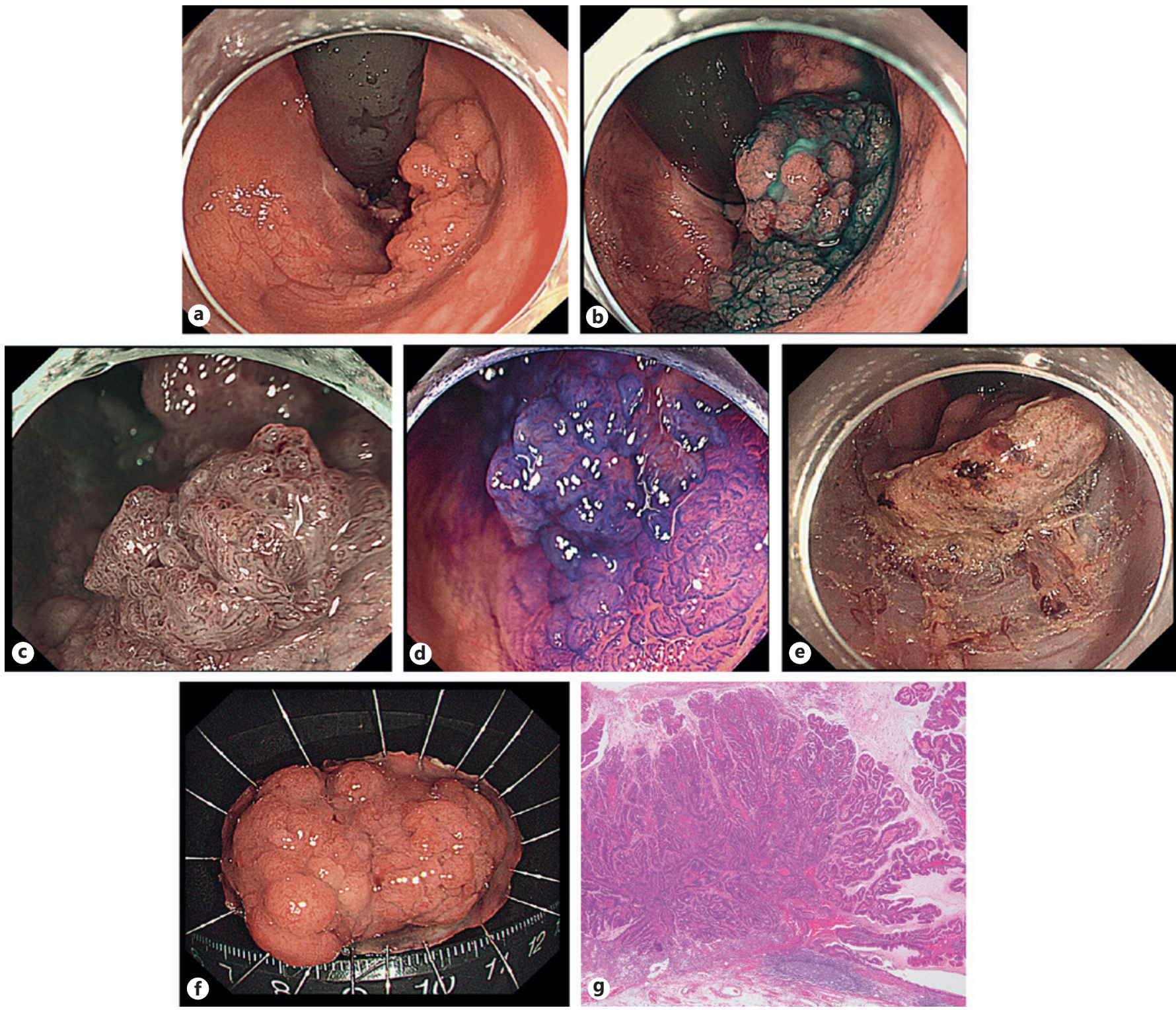

Fig. 1. A rectal cancer effectively treated by ESD for therapeutic purposes. a A laterally spreading lesion of $35 \mathrm{~mm}$ in diameter was found in the rectum. b Chromoendoscopic view stained with indigo carmine. c Endoscopic view with NBI. The capillary pattern was Type $2 \mathrm{~B}$ in the JNET classification. $\mathbf{d}$ The pit pattern in crystal violet staining was Type $\mathrm{V}_{\mathrm{I}}$-low in the Kudo classification. e Severe fibrosis was observed in the submucosal layer. $f$ Macroscopic view

cer (high-grade dysplasia). Although severe fibrosis was recognized in the submucosal layer during ESD, additional injection into the submucosal layer was effective in dilating a space between the mucosa and muscle layer (Fig. 2). Histological examination of the resected specimen revealed well-differentiated adenocarcinoma (submucosal invasion $200 \mu \mathrm{m}$ ) with lymphatic involvement.

of the resected specimen. $\mathbf{g}$ Histological examination of the resected tissue revealed mucosal well-differentiated adenocarcinoma without lymphatic or vascular involvement. The vertical and horizontal margins were both negative. ESD, endoscopic submucosal dissection; NBI, narrow-band imaging; JNET, Japanese NBI Expert Team.

The vertical and horizontal margins were both negative. The resection was assessed as non-curative, so the patient underwent restorative proctocolectomy as an additional surgery. The pathological results showed no residual carcinoma, no other dysplastic lesions at other sites of the resected specimen, and no lymph node metastasis. 

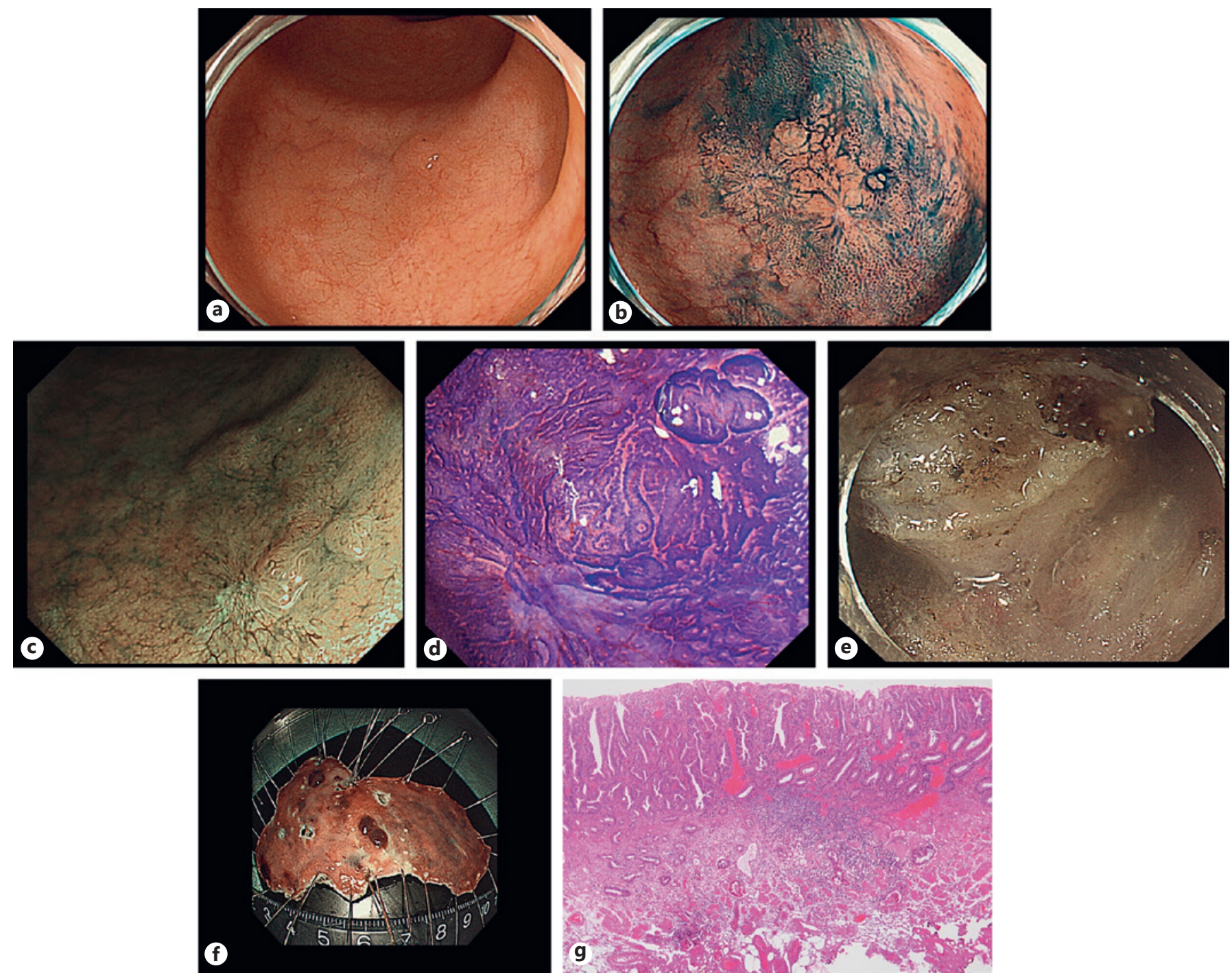

Fig. 2. A rectal cancer effectively examined by ESD for diagnostic purposes. a A laterally spreading lesion of $11 \mathrm{~mm}$ in diameter was found in the rectum. b Chromoendoscopic view stained with indigo carmine. c Endoscopic view with NBI. The capillary pattern was Type $2 \mathrm{~B}$ in the JNET classification. $\mathbf{d}$ The pit pattern in crystal violet staining was Type $\mathrm{V}_{\mathrm{I}^{-}}$low in the Kudo classification. e Severe fibrosis was observed in the submucosal layer. $f$ Macro-

\section{Conclusion}

Physicians engaged in the management of ulcerative colitis-related lesions seem to have reached a consensus on the acceptability of endoscopic resection for circumscribed areas of dysplasia followed by close endoscopic surveillance. ESD for neoplasms in ulcerative colitis is feasible. ESD for circumscribed dysplasia in ulcerative colitis can spare patients from unnecessary surgery. scopic view of the resected specimen. $\mathbf{g}$ Histological examination of the resected tissue revealed a well-differentiated adenocarcinoma (submucosal invasion $200 \mu \mathrm{m}$ ) with lymphatic involvement. The vertical and horizontal margins were both negative. ESD, endoscopic submucosal dissection; NBI, narrow-band imaging; JNET, Japanese NBI Expert Team. 
could also enhance the safety of therapeutic endoscopy [23-25]. No adequate assessment has been reported, however, on the long-term outcomes of ESD for ulcerative colitis. A further accumulation of long-term results is awaited.

\section{References}

1 Löfberg R, Brostrom O, Karlen P, Tribukait B, Ost A: Colonoscopic surveillance in longstanding total ulcerative colitis - a 15-year follow-up study. Gastroenterology 1990;99: 1021-1031.

2 Ekbom A, Helmick C, Zack M, Adami HO: Ulcerative colitis and colorectal cancer. a population-based study. N Engl J Med 1990;323: 1228-1233.

3 Bernstein CN, Shanahan F, Weinstein WM: Are we telling patients the truth about surveillance colonoscopy in ulcerative colitis? Lancet 1994;343:71-74.

4 Lennard-Jones JE, Melville DM, Morson BC, Ritchie JK, Williams CB: Precancer and cancer in extensive ulcerative colitis: findings among 401 patients over 22 years. Gut 1990; 31:800-806.

5 Leidenius M, Kellokumpu I, Husa A, Riihela M, Sipponen P: Dysplasia and carcinoma in longstanding ulcerative colitis: an endoscopic and histological surveillance programme. Gut 1991;32:1521-1525.

6 Rutter MD, Riddell RH: Colorectal dysplasia in inflammatory bowel disease: a clinicopathologic perspective. Clin Gastroenterol Hepatol 2014;12:359-367.

7 Hurlstone DP, Sanders DS, Atkinson R, et al: Endoscopic mucosal resection for flat neoplasia in chronic ulcerative colitis: can we change the endoscopic management paradigm? Gut 2007;56:838-846.

8 Iacucci M, Uraoka T, Fort Gasia M, Yahagi N: Novel diagnostic and therapeutic techniques for surveillance of dysplasia in patients with inflammatory bowel disease. Can J Gastroenterol Hepatol 2014;28:361-370.

\section{Disclosure Statement}

The authors declare no conflicts of interest for this article.
9 Laine L, Kaltenbach T, Barkun A, McQuaid KR, Subramanian V, Soetikno R; SCENIC Guideline Development Panel: SCENIC international consensus statement on surveillance and management of dysplasia in inflammatory bowel disease. Gastroenterology 2015;148:639-651.e28.

10 Mowat C, Cole A, Windsor A, et al: Guidelines for the management of inflammatory bowel disease in adults. Gut 2011;60:571-607.

11 Nakajima T, Saito Y, Tanaka S, et al: Current status of endoscopic resection strategy for large, early colorectal neoplasia in Japan. Surg Endosc 2013;27:3262-3270.

12 East JE, Toyonaga T, Suzuki N: Endoscopic management of nonpolypoid colorectal lesions in colonic IBD. Gastrointest Endosc Clin N Am 2014;24:435-445.

13 Uraoka T, Parra-Blanco A, Yahagi N: Colorectal endoscopic submucosal dissection in Japan and Western countries. Dig Endosc 2012; 24(suppl 1):80-83.

14 Iacopini F, Saito Y, Yamada M, et al: Curative endoscopic submucosal dissection of large nonpolypoid superficial neoplasms in ulcerative colitis (with videos). Gastrointest Endosc 2015;82:734-738.

15 Suzuki N, Toyonaga T, East JE: Endoscopic submucosal dissection of colitis-related dysplasia. Endoscopy 2017;49:1237-1242.

16 Kinoshita S, Uraoka T, Nishizawa T, et al: The role of colorectal endoscopic submucosal dissection in patients with ulcerative colitis. Gastrointest Endosc 2018;87:1079-1084.

17 Petrelli NJ, Letourneau R, Weber T, Nava ME, Rodriguez-Bigas M: Accuracy of biopsy and cytology for the preoperative diagnosis of colorectal adenocarcinoma. J Surg Oncol 1999;71:46-49.
18 Kinoshita S, Nishizawa T, Ochiai Y, et al: Accuracy of biopsy for the preoperative diagnosis of superficial nonampullary duodenal adenocarcinoma. Gastrointest Endosc 2017;.86: 329-332.

19 Goto N, Kusaka T, Tomita Y, et al: Magnifying narrow-band imaging with acetic acid to diagnose early colorectal cancer. World J Gastroenterol 2014;20:16306-16310.

20 Asayama N, Oka S, Tanaka S, Hayashi N, Arihiro K, Chayama K: Endoscopic submucosal dissection as total excisional biopsy for clinical T1 colorectal carcinoma. Digestion 2015; 91:64-69.

21 Fujimoto A, Goto O, Nishizawa T, et al: Gastric ESD may be useful as accurate staging and decision of future therapeutic strategy. Endosc Int Open 2017;5:E90-E95.

22 Pioche M, Rivory J, Nishizawa T, et al: Randomized comparative evaluation of endoscopic submucosal dissection self-learning software in France and Japan. Endoscopy 2016;48:1076-1083.

23 Yahagi N, Nishizawa T, Akimoto T, Ochiai Y, Goto O: New endoscopic suturing method: string clip suturing method. Gastrointest Endosc 2016;84:1064-1065.

24 Tashima T, Ohata K, Sakai E, et al: Efficacy of an over-the-scope clip for preventing adverse events after duodenal endoscopic submucosal dissection: a prospective interventional study. Endoscopy 2018;50:487-496.

25 Mori H, Kobara H, Nishiyama N, Masaki T: Current status and future perspectives of endoscopic full-thickness resection. Dig Endosc 2018;30(suppl 1):25-31. 